\title{
28 Research Square \\ ICU-Acquired Pneumonia is a Risk Factor of a Poor Health Post-Covid-19 Syndrome
}

Ignacio Martin-Loeches

Saint James's Hospital https://orcid.org/0000-0002-5834-4063

Anna Motos

Hospital Clinic Universitario

Rosario Menéndez

La Fe University and Polytechnic Hospital: Hospital Universitari i Politecnic La Fe

Albert Gabarrus

Clinico

Jessica González

UdL: Universitat de Lleida

Laia Fernández-Barat

Hospital of Clinic

Adrián Ceccato

Clínica Girona: Clinica Girona

Raquel Pérez

UBS

Dario García-Gasulla

BSC: Bismarck State College

Ricard Ferrer

Vall d'Hebron Hospital: Vall d'Hebron Hospital Universitari

Jordi Riera

Vall d'Hebron Hospital: Vall d'Hebron Hospital Universitari

José Ángel Lorente

Getafe University Hospital: Hospital Universitario de Getafe

\section{Oscar Peñuelas}

Getafe University Hospital: Hospital Universitario de Getafe

Jesús $\mathrm{F}$ Bermejo-Martin

EOI de Valladolid: Escuela Oficial de Idiomas de Valladolid

David de Gonzalo-Calvo

UdL: Universitat de Lleida

\section{Alejandro Rodríguez}

Joan XXIII University Hospital in Tarragona: Hospital Universitari de Tarragona Joan XXIII

Ferran Barbé 
UdL: Universitat de Lleida

Luciano Aguilera

osadieka

Rosario Amaya Villar

Virgen Macarena University Hospital: Hospital Universitario Virgen Macarena

Carme Barberà

UdL: Universitat de Lleida

José Barberán

madrid

Aaron Blandino Ortiz

Madrid-

Elena Bustamante-Munguira

castilla

Jesús Caballero

UdL: Universitat de Lleida

Cristina Carbajales

sergas

Nieves Carbonell

UdL: Universitat de Lleida

Mercedes Catalán-González

madrid

Cristóbal Galbán

SERGAS: Servicio Galego de Saude

Víctor D. Gumucio Sanguino

Bellvitge University Hospital: Hospital Universitari de Bellvitge

Maria del Carmen de la Torre

Hospital de Mataró: Hospital de Mataro

Emili Díaz

Parc Tauli Foundation-UAB University Institute: Fundacio Parc Tauli

Elena Gallego

UdL: Universitat de Lleida

José Luis García Garmendia

UdL: Universitat de Lleida

José Garnacho-Montero

Virgen del Rocio University Hospital: Hospital Universitario Virgen del Rocio

José M. Gómez

madrid

Ruth Noemí Jorge García

UdL: Universitat de Lleida 


\section{Ana Loza-Vázquez}

vallme

Judith Marin-Corral

Maroondah Hospital

Amalia Martínez de la Gándara

UdL: Universitat de Lleida

Ignacio Martínez Varela

UdL: Universitat de Lleida

Juan Lopez Messa

UdL: Universitat de Lleida

\section{Guillermo M Albaiceta}

SESPAS: Ministerio de Salud Publica y Asistencia Social

\section{Mariana Andrea Novo}

UdL: Universitat de Lleida

\section{Yhivian Peñasco}

scsalud

\section{Pilar Ricart}

UdL: Universitat de Lleida

\section{Luis Urrelo-Cerrón}

Hospital Verge de la Cinta

\section{Angel Sánchez-Miralles}

coma

\section{Susana Sancho Chinesta}

PESSE: PES University - Electronic City Campus

\section{Lorenzo Socias}

IVI Mallorca

\section{Jordi Solé-Violan}

HUGC Dr Negrin: Hospital Universitario de Gran Canaria Dr Negrin

\section{Luis Tamayo Lomas}

Valladolid University Hospital: Hospital Clinico Universitario de Valladolid

\section{Pablo Vidal}

SERGAS: Servicio Galego de Saude

Antoni Torres ( $\sim$ ATORRES@clinic.cat )

Universitat de Barcelona https://orcid.org/0000-0002-5470-2978

\section{Research}

Keywords: COVID-19, CT abnormalities, ICU, lung function, SARS-CoV-2, sequelae, Post-COVID. 
Posted Date: September 13th, 2021

DOl: https://doi.org/10.21203/rs.3.rs-885672/v1

License: (c) (i) This work is licensed under a Creative Commons Attribution 4.0 International License. Read Full License 


\section{Abstract}

Background. Some patients who had previously presented with COVID-19 have been reported to develop persistent COVID-19 symptoms. Whilst this information has been adequately recognised and extensively published with respect to non-critically ill patients, less is known about the prevalence and risk factors and characteristics of persistent COVID_19. On other hand these patients have very often intensive care unit-acquired pneumonia (ICUAP). A second infectious hit after COVID increases the length of ICU stay and mechanical ventilation and could have an influence in the poor health post-Covid 19 syndrome in ICU discharged patients

Methods: This prospective, multicentre and observational study was done across 40 selected ICUs in Spain. Consecutive patients with COVID-19 requiring ICU admission were recruited and evaluated three months after hospital discharge.

Results: A total of 1,255 ICU patients were scheduled to be followed up at 3 months; however, the final cohort comprised 991 (78.9\%) patients. A total of 315 patients developed ICUAP ( $97 \%$ of them had ventilated ICUAP) Patients requiring invasive mechanical ventilation had persistent, post-COVID-19 symptoms than those who did not require mechanical ventilation. Female sex, duration of ICU stay, and development of ICUAP were independent risk factors for persistent poor health post-COVID-19.

Conclusions: Persistent, post-COVID-19 symptoms occurred in more than two-thirds of patients. Female sex, duration of ICU stay and the onset of ICUAP comprised all independent risk factors for persistent poor health post-COVID-19. Prevention of ICUAP could have beneficial effects in poor health post-Covid 19

\section{Introduction}

Clinical presentation of severe acute respiratory syndrome coronavirus 2 (SARS-CoV-2) infection ranges from mild to severe[1]. Disease severity, including refractory acute respiratory failure (ARF) and acute respiratory distress syndrome (ARDS), may require admission to an intensive care unit (ICU) [2]. Patients often need invasive mechanical ventilation and can develop multiorgan failure[3]. Duration of ICU stay is long amongst survivors, and mortality can be high in patients with ARDS, reaching between $40-50 \%[4]$. On the other hand ICU admitted patients, especially those requiring mechanical ventilation, have a high prevalence of other infections complications such as ICU acquired pneumonia (ICUAP) [5]that could have a role in the persistence of symptoms after discharge Additionally, although often sufficiently recovered for hospital discharge, patients with either mild or severe disease are at risk of developing a condition known as persistent poor health post-COVID-19, post-COVID syndrome or long COVID[6][7][8].

Currently, no consensus definition for the symptoms of poor health post-COVID-19 exist; however, the most common symptoms include fatigue, shortness of breath, weakness and asthenia[9]. Some publications have reported a high prevalence of persistent poor health post-COVID-19. Specifically, fatigue and dyspnoea were the most frequent symptoms, and their prevalence and intensity were not 
correlated with initial disease severity [10]. With respect to information regarding persistent poor health post-COVID-19 in patients that have survived a stay in the ICU, little remains known[11][12][13]. Very importantly risk factors that are present in the acute period of the disease are not well known. As pointed out in a recent position manuscript the recognition of risk factors during the acute period is a research priority to understand the long-term sequalae of COVID-19[14].

In the present manuscript, we analysed the poor health post-COVID-19 in the initial cases of hospitalised patients with COVID-19 at 3-month follow-up since hospital discharge. We hypothesised that critically ill patients presented with a high, persistent post-COVID-19 symptoms and significant abnormalities in both lung function tests and radiology. Herein, we summarised poor health post-COVID-19, functional respiratory parameters and radiologic features of patients discharged from the ICU at the 3-month followup. The main objective of this study was to determine the risk factors of the acute period associated with

poor health post-COVID-19 in ICU survivors at the 3-month follow-up since hospital discharge and identify risk factors associated with poor recovery. We also aimed to analyse lung function and radiologic abnormalities in critically ill patients after hospital discharge.

\section{Material And Methods}

Study design and population

We carried out a prospective, multicentre and observational study at 40 selected ICUs in Spain with critically ill patients initially admitted after 16 February 2020. We consecutively recruited patients with COVID-19 requiring ICU admission and performed a follow-up at three months since hospital discharge. This study is a pre-planned analysis of the ongoing multicentre study called CIBERESUCICOVID (ClinicalTrials.gov Identifier: NCT04457505). We then asked staff from each centre to prospectively obtain data for ICU-admitted patients aged 18 years or older with positive polymerase chain reactions (PCR) for SARS-CoV-2. Re-admitted patients and previously tracheostomised patients were not included. The study received approval by the institution's Internal Review Board (Comité Ètic d'Investigació Clínica, registry number $\mathrm{HCB} / 2020 / 0370$ ). We obtained informed consent for most patients by using emergency consent mechanisms in accordance with ethics approval guidelines for the study. Further participating centres either received ethics approval from their institutions or had waived ethics approval. Finally, we de-identified all clinical data to allow for the waiver of informed consent.

\section{Data collection}

Recorded data included demographic characteristics, comorbidities, time course of illness, treatments administered, laboratory and microbiologic data, radiologic findings on chest x-rays, CT scans, ventilatory parameters in patients with invasive mechanical ventilation, complications during ICU stay, and outcomes. We determined disease severity and assessed organ failure using the Sequential Organ Failure Assessment (SOFA) score, calculating both within the first day of ICU admission[15]. Ventilatory management strategies were not standardised amongst centres and were left to the discretion of the attending clinician, based on National Ministry of Health recommendations, and supported by 
international guidelines. We defined ICU-acquired pneumonia (ICUAP) as pneumonia developing in patients in the ICU for $\geq 48$ hours. Basis of ICUAP diagnosis comprised new or progressive radiologic pulmonary infiltrates together with at least two of the subsequent characteristics: temperature $>38^{\circ} \mathrm{C}$ or $<$ $36^{\circ}$ C; leucocytosis $>12,000 / \mathrm{mm} 3$ or leucopoenia $<4,000 / \mathrm{mm} 3$; or purulent respiratory secretion $[5,16]$. We confirmed an ARDS diagnosis using the Berlin definition[17].

\section{Procedures}

Poor health post-COVID-19 was defined by the presence of any of the following symptoms: dyspnoea, weakness, asthenia, myalgia, cough, numbness, headache, anosmia and ageusia. We provided electronic case report forms using a secure website. For all patients, we recorded demographic characteristics, duration of ICU and hospital stays, the McCabe classification of comorbidities and likelihood of survival (likely to survive 5 years, $1-5$ years [ultimately fatal], or $<1$ year [rapidly fatal]), the SOFA score to predict hospital mortality, and outcome (ICU mortality). We calculated static compliance of the respiratory system as tidal volume/(end-inspiratory plateau pressure-total PEEP). Chest CT scans and CT pulmonary angiograms were obtained when clinically indicated and technically feasible. The Modified Medical Research Council (mMRC) dyspnoea scale grades the impact of dyspnoea on daily activities throughout the prior seven days and thereby quantifies the disability or physical limitations associated with dyspnoea [18]. Finally, to aid analysis, we clustered patients into groups according to clinical resolution.

\section{Outcomes}

The primary outcome of our study was to determine the risk factors and prevalence of poor health postCOVID-19 in critically ill patients per clinical presentation at three months of hospital discharge. The secondary outcome included determining associations between poor health post-COVID-19 symptoms and abnormalities in lung function tests, radiologic characteristics, and laboratory parameters.

\section{Statistical analysis}

The study sample size was not formally calculated but instead based on the nature of the study and preplanned dates. We used SPSS (version 20) for data analysis. All p values were two-tailed. We considered differences as significant if $p$ was less than $0 \cdot 05$. We reported categorical variables as numbers and frequencies (\%), normally distributed continuous variables as means (standard deviation [SD]), and skewed continuous variables as medians ((interquartile range [IQR]. We performed both $\chi 2$ tests or Fisher's exact test to compare qualitative variables and Student's $t$ tests and ANOVAs or Mann-Whitney $U$ and non-parametric Kruskal-Wallis tests to compare normally distributed or skewed continuous variables, whenever appropriate. We undertook univariate analyses of predictors of post-covid syndrome, using the $\chi 2$ test and Student's $t$ test. We entered all variables from the univariate analysis with $p$ values below $0 \cdot 1$ or which were clinically relevant into regression analyses as potential predictor variables. We made two adjustments to determine which risk factors were associated with poor health post-COVID-19 and possible confounders: 1) we calculated adjusted estimates of the effect size and corresponding $95 \%$ 
confidence intervals (Cls) using a multivariable logistic regression model(backward stepwise selection model) and 2) to account for centre effects, we performed a mixed-effects multivariable model for sensitivity analysis, as defined by a binomial probability distribution and a logit link function, and centres as a random effect.

\section{Results}

Definition of the population

We monitored patients after hospital discharge for a median of 77 [57-99] days. A total of 1,255 patients were scheduled to be followed up for three months. However, we could not perform a 3-month follow-up of 264 patients. The final cohort comprised 991 (78.9\%) patients. A total of 731 (73.8\%) patients developed persistent post-COVID-19 symptoms. A flow chart with the percentage of patients with persistent post-COVID-19 symptoms are displayed in Fig. 1. Patient characteristics are shown in Table 1. 
Table 1

Patient characteristics of the population enrolled in the study based on persistent post-COVID-19 symptoms at 3-month follow-up.

\begin{tabular}{|ll|}
\hline Demographic item & $n$ \\
\hline Age*, mean (SD), years & $58.2(\mathrm{SD} 12.3)$ \\
\hline Sex (Female), n (\%) & $405(32.3)$ \\
\hline SOFA*, mean (SD) & $4.6(\mathrm{SD} 2.8)$ \\
\hline CHF, n (\%) & $118(9.5)$ \\
\hline Hypertension), n (\%) & $552(44.2)$ \\
\hline COPD), n (\%) & $100(8.0)$ \\
\hline Asthma), n (\%) & $79(6.3)$ \\
\hline CKD), n (\%) & $69(5.5)$ \\
\hline Cirrhosis ), n (\%) & $15(1.2)$ \\
\hline Mild liver failure), n (\%) & $27(2.2)$ \\
\hline Neurologic), n (\%) & $66(1.8)$ \\
\hline Dementia), n (\%) & $6(0.2)$ \\
\hline Autoimmune), n (\%) & $71(5.7)$ \\
\hline Gastrointestinal), n (\%) & $100(8.1)$ \\
\hline Endocrine), n (\%) & $90(7.2)$ \\
\hline Obesity (BMl > 30 kg/m²), n (\%) & $483(38.7)$ \\
\hline Diabetes Mellitus ), n (\%) & $247(19.8)$ \\
\hline Haematologic disease), n (\%) & $73(5.8)$ \\
\hline Solid cancer), n (\%) & $37(3.0)$ \\
\hline Transplant), n (\%) & $12(0.3)$ \\
\hline HIV), n (\%) & $8(0.6)$ \\
\hline
\end{tabular}

Abbreviations: SOFA: Sequential Organ Failure Assessment. CHF: congestive heart failure. COPD: Chronic obstructive pulmonary disease. CKD: Chronic kidney disease. BMI: Body mass index. HIV: Human immunodeficiency virus. NIVM: Non-invasive mechanical ventilation. iMV: Invasive mechanical ventilation. ICU: Intensive care unit. ECMO: Extracorporeal membrane oxygenation. CRRT: Continuous renal replacement therapy. NMB: Neuromuscular blockade. CPR: Cardiopulmonary resuscitation. ICUAP: Intensive care unit-acquired pneumonia. ARDS: Acute respiratory distress syndrome. *Mean and SD 


\begin{tabular}{|c|c|}
\hline Demographic item & n \\
\hline Alcohol), n (\%) & $32(2.6)$ \\
\hline Oxygen requirement), n (\%) & 1228(99.5) \\
\hline NIV), n (\%) & $440(35.9)$ \\
\hline iMV), n (\%) & $858(69.0)$ \\
\hline Prone), n (\%) & 717(57.6) \\
\hline Tracheostomy ), n (\%) & $378(30.6)$ \\
\hline ICU length of stay),, mean (SD), days & $20.1(18.2)$ \\
\hline ECMO), n (\%) & $24(1.9)$ \\
\hline CRRT), n (\%) & $62(5.0)$ \\
\hline Shock), n (\%) & $756(20.1)$ \\
\hline NMB), n (\%) & 706(57.3) \\
\hline Corticosteroids), n (\%) & $943(25.1)$ \\
\hline CPR), n (\%) & $10(0.8)$ \\
\hline ICUAP), n (\%) & $315(25.5)$ \\
\hline ARDS), n (\%) & $955(77.3)$ \\
\hline NTX), n (\%) & $58(4.7)$ \\
\hline COP), n (\%) & $52(4.2)$ \\
\hline PE), n (\%) & 108(8.7) \\
\hline Delirium), n (\%) & $301(24.4)$ \\
\hline \multicolumn{2}{|c|}{$\begin{array}{l}\text { Abbreviations: SOFA: Sequential Organ Failure Assessment. CHF: congestive heart failure. COPD: } \\
\text { Chronic obstructive pulmonary disease. CKD: Chronic kidney disease. BMI: Body mass index. HIV: } \\
\text { Human immunodeficiency virus. NIVM: Non-invasive mechanical ventilation. iMV: Invasive } \\
\text { mechanical ventilation. ICU: Intensive care unit. ECMO: Extracorporeal membrane oxygenation. CRRT } \\
\text { Continuous renal replacement therapy. NMB: Neuromuscular blockade. CPR: Cardiopulmonary } \\
\text { resuscitation. ICUAP: Intensive care unit-acquired pneumonia. ARDS: Acute respiratory distress } \\
\text { syndrome. *Mean and SD }\end{array}$} \\
\hline
\end{tabular}

General assessment and hospital burden

We performed the mMRC dyspnoea scale in 402 patients, of whom $59 \%$ were grade $1 ; 28.4 \%$, grade 2 ; $10 \%$, grade 3 ; and $2.2 \%$, grade 4 . Physical examination of patients revealed crackles in $8.2 \%(n=82)$ and showed a trend of its presence in patients with persistent post-COVID-19 symptoms $(9.9 \%$ vs $5.8 \%, \mathrm{p}=$ 0.05). After discharge, 33 (3.2\%) patients presented with infectious complications; $172(15.1 \%)$ attended the emergency department; and $63(5 \%)$ were readmitted to hospital. No significant differences were 
observed in patients with or without persistent post-COVID-19 symptoms with respect to follow-up clinic visits $(63.5 \%$ vs $59.6 \%$, p 0.3). Patients with persistent post-COVID-19 symptoms required additional respiratory therapy after hospital discharge (Supplementary Table 1).

Clinical features and associations with functional (lung function tests), imaging and laboratory results at 3-month follow-up

We performed a radiologic work-up in $471(47.5 \%)$ patients. A similar number of patients with or without persistent post-COVID-19 symptoms underwent either chest radiography $(51.4 \%$ vs $48.1 \%, \mathrm{p} 0.3)$ or chest CT scans $(40.5 \%$ vs $45.9 \%, p=0.1)$. Persistently abnormal chest $x$-rays were observed in $21.4 \%$ of patients. Patients with persistent post-COVID-19 symptoms more frequently had abnormal x-rays and chest CT scans showing diffuse interstitial lung patterns than those without such symptoms. Patients who required invasive mechanical ventilation more often presented interstitial lung disease patterns in chest CT scans. Further details are shown in Table 2. 
Table 2

Chest imaging and lung function tests in patients with persistent post-COVID-19 symptoms and in those who underwent invasive mechanical ventilation at 3-month follow-up.

\begin{tabular}{|c|c|c|c|c|c|c|}
\hline & \multicolumn{3}{|l|}{ Post-COVID } & \multicolumn{3}{|c|}{ Invasive mechanical ventilation } \\
\hline & Yes & No & $\mathrm{p}$ & Yes & No & $P$ value \\
\hline \multicolumn{7}{|l|}{ Chest x-ray, n (\%) } \\
\hline Abnormal & $597(81.7)$ & 182(70) & $<0.01$ & 715(83.3) & $294(76.2)$ & 0.004 \\
\hline ILD & $2(0.8)$ & $5(0.7)$ & 0.5 & $5(0.6)$ & $2(0.5)$ & 0.9 \\
\hline Persistent infiltrates & $34(13.1)$ & $130(17.8)$ & 0.08 & $129(15)$ & $46(11.9)$ & 0.1 \\
\hline Fibrotic tract & $12(4.6)$ & $54(7.4)$ & 0.1 & $50(5.8)$ & $19(4.9)$ & 0.5 \\
\hline Emphysema & 0 & $3(0.4)$ & 0.5 & $3(0.3)$ & 0 & 0.5 \\
\hline \multicolumn{7}{|l|}{ CT scan, n (\%) } \\
\hline Abnormal & 685(93.7) & 238(91.5) & 0.2 & $810(94.4)$ & $364(94.3)$ & 0.9 \\
\hline ILD & 0 & $18(2.5)$ & 0.006 & $6(1.6)$ & $12(1.4)$ & 0.8 \\
\hline Persistent infiltrates & $43(16.5)$ & 118(16.1) & 0.9 & 131(15.3) & $42(10.9)$ & 0.04 \\
\hline Fibrotic & $27(10.4)$ & $88(12)$ & 0.5 & $88(10.3)$ & $35(9.1)$ & 0.5 \\
\hline Emphysema & $4(1.5)$ & $23(3.1)$ & 0.2 & $20(2.3)$ & $7(1.8)$ & 0.5 \\
\hline PE, n (\%) & $2(0.8)$ & $11(1.5)$ & 0.2 & $12(1.4)$ & $1(0.3)$ & 0.07 \\
\hline \multicolumn{7}{|l|}{ LFTs, n (\%) } \\
\hline FEV1 & $94.2(18.2)$ & $87.5(18.1)$ & $<0.01$ & 89.3(19.1) & $88.1(17.1)$ & 0.4 \\
\hline FEV1/FVC & $91.4(16.3)$ & $97.2(14.6)$ & $<0.01$ & $96.1(14.9)$ & $96.8(15.8)$ & 0.5 \\
\hline $\mathrm{DLCO}(\mathrm{mL} / \mathrm{min} / \mathrm{mm} \mathrm{Hg})$ & 78.3(17.4) & $67.1(17.7)$ & $<0.01$ & $67.0(18.1)$ & $74.1(17.4)$ & $<0.01$ \\
\hline DLCO 80 & 205(78.8) & $459(62.8)$ & $<0.01$ & $626(73)$ & $280(72.5)$ & 0.4 \\
\hline $\begin{array}{l}\text { Data are mean (SD) or nu } \\
\text { tomography. ILD: Diffuse } \\
\text { FEV1: Forced expiratory } \\
\text { capacity for carbon mon }\end{array}$ & $\begin{array}{l}\text { ber of patie } \\
\text { terstitial lun } \\
\text { ume in the ff } \\
\text { de. }\end{array}$ & $\begin{array}{l}\text { S (\%). Abbre } \\
\text { disease. PE: } \\
\text { t second. Fi }\end{array}$ & $\begin{array}{l}\text { ations: S } \\
\text { ulmonar } \\
\text { : Forced }\end{array}$ & $\begin{array}{l}\text { : Standard o } \\
\text { embolism. } \\
\text { ital capacity }\end{array}$ & $\begin{array}{l}\text { viation. CT: } \\
\text { :T: Lung fun } \\
\text { DLCO: Diffus }\end{array}$ & $\begin{array}{l}\text { mputed } \\
\text { ion test. } \\
\text { ig }\end{array}$ \\
\hline
\end{tabular}

We performed lung function tests (LFT) in 535 (53.9\%) patients and diffusing capacity of the lungs for carbon monoxide (DLCO) in $464(46.8 \%)$. LFTs ( $57.1 \%$ vs $49 \%, p=0.03)$ were performed more often in patients with persistent post-COVID-19 symptoms, whilst there were no significant differences in performing DLCO between patients with or without persistent post-COVID-19 symptoms $(50.7 \%$ vs $55.3 \%$, $p=0.2)$. A total of $665(67.4 \%)$ patients underwent invasive mechanical ventilation. Patients with invasive mechanical ventilation had a higher percentage of persistent post-COVID-19 symptoms than those 
without mechanical ventilation $(70.2 \%$ vs $59.6 \%, p=0.003)$. Table 3 shows invasive mechanical ventilator and oxygenation parameters obtained sequentially at days 1 and 3 after the initiation of invasive mechanical ventilation. Only pulmonary compliance calculated at the time of initiation of mechanical ventilation and the value of $\mathrm{PaO} 2 / \mathrm{FiO}_{2}$ at day 3 were significantly worse in patients with persistent postCOVID-19 symptoms. 
Table 3

Respiratory parameters in patients with invasive mechanical ventilation based on persistent post-COVID-

19 symptoms at 3-month follow-up

\begin{tabular}{|c|c|c|c|c|}
\hline & \multicolumn{4}{|c|}{ Post-COVID } \\
\hline Variable & Time-point & No & Yes & P-value \\
\hline \multirow[t]{3}{*}{ PaO2/FiO2, mean (SD) } & Intubation & $130.5(73.4)$ & $131.6(74.9)$ & 0.8 \\
\hline & Day 3 & $192.0(85.7)$ & $180.0(75.0)$ & 0.1 \\
\hline & Change from intubation & $66.3(100.8)$ & $49.0(111.7)$ & 0.1 \\
\hline \multirow[t]{3}{*}{$\mathrm{PaCO} 2$, mean (SD), mmHg } & Intubation & $39.4(9.5)$ & $40.4(10.6)$ & 0.2 \\
\hline & Day 3 & $43.0(9.4)$ & $44.8(9.8)$ & 0.03 \\
\hline & Change from intubation & $3.4(11.5)$ & $3.4(12.2)$ & 1.0 \\
\hline \multirow[t]{3}{*}{ VT admission, mean (SD), mL } & Intubation & $7.1(1.2)$ & $6.9(3.0)$ & 0.5 \\
\hline & Day 3 & $7.2(1.2)$ & $7.1(3.1)$ & 0.7 \\
\hline & Change from intubation & $0.2(1.0)$ & $0.3(1.6)$ & 0.6 \\
\hline \multirow[t]{3}{*}{ PEEP, mean (SD), cmH2O } & Intubation & $12.5(2.5)$ & $12.4(2.5)$ & 0.7 \\
\hline & Day 3 & $12.0(2.7)$ & $12.1(2.7)$ & 0.5 \\
\hline & Change from intubation & $-0.6(2.8)$ & $-0.4(2.9)$ & 0.6 \\
\hline \multirow[t]{3}{*}{$\Delta \mathrm{P}$, mean (SD) } & Intubation & $10.8(4.6)$ & $11.8(4.7)$ & 0.1 \\
\hline & Day 3 & $10.7(4.2)$ & $11.8(4.8)$ & 0.2 \\
\hline & Change from intubation & $-0.6(3.0)$ & $-0.1(5.1)$ & 0.7 \\
\hline \multirow[t]{3}{*}{ Compliance, mean (SD) } & Intubation & $66.3(86.4)$ & $47.9(35.9)$ & 0.02 \\
\hline & Day 3 & $42.6(79.0)$ & $46.0(29.0)$ & 0.6 \\
\hline & Change from intubation & $-26.1(127.4)$ & $0.2(43.0)$ & 0.1 \\
\hline \multirow[t]{3}{*}{ VR, mean (SD) } & Intubation & $1.7(0.5)$ & $1.7(0.5)$ & 0.7 \\
\hline & Day 3 & $1.8(0.5)$ & $1.8(0.5)$ & 0.8 \\
\hline & Change from intubation & $0.2(0.5)$ & $0.2(0.6)$ & 0.7 \\
\hline \multicolumn{5}{|c|}{$\begin{array}{l}\text { Data are mean (SD). Abbreviations: VT: Tidal volume. } \triangle \mathrm{P} \text { : Driving pressure PEEP: Positive end- } \\
\text { expiratory pressure. VR: Ventilatory ratio. The delta measurements were computed as the difference in } \\
\text { amplitude between day } 3 \text { of initiation of invasive mechanical ventilation and day } 1 \text { of initiation of } \\
\text { invasive mechanical ventilation. }\end{array}$} \\
\hline
\end{tabular}

Laboratory data at day 1 of ICU admission are detailed in Table 4. No significant differences were observed in most parameters; however, fibrinogen was significantly higher in patients with persistent 
post-COVID-19 symptoms.

Table 4

Laboratory parameters in patients with invasive mechanical ventilation based on persistent post-COVID19 symptoms at 3-month follow-up.

\begin{tabular}{|c|c|c|c|}
\hline & \multicolumn{3}{|l|}{ Post-COVID } \\
\hline & No & Yes & $P$ value \\
\hline & Value (SD) & Mean & \\
\hline Haemoglobin, mean (SD), g/dL & $14.7(11.3)$ & $13.9(1.5)$ & 0.3 \\
\hline WCC, mean (SD), x 109/L & 7.3(2.2) & $6.81(2.3)$ & 0.06 \\
\hline Lymphocytes, mean (SD), x 109/L & $2.2(0.9)$ & $2.3(1.4)$ & 0.6 \\
\hline Neutrophiles, mean (SD), x 109/L & $4.4(3.6)$ & $3.7(1.9)$ & 0.09 \\
\hline Monocytes, mean (SD), x 109/L & $0.6(0.5)$ & $0.5(0.2)$ & 0.2 \\
\hline Haematocrit, mean (SD), x 109/L & $41.4(5.2)$ & $42.1(4.6)$ & 0.1 \\
\hline Platelets, mean (SD), x 109/L & $259.3(73.5)$ & $247.4(88.7)$ & 0.2 \\
\hline Prothrombin time, mean (SD), sec & $11.9(6.8)$ & $11.8(4.1)$ & 0.9 \\
\hline INR, mean (SD), IU & $1.3(0.6)$ & $1.1(0.3)$ & 0.5 \\
\hline D-dimer, mean (SD), ng/mL & $410.2(445.1)$ & 433.2(393.4) & 0.6 \\
\hline Fibrinogen, mean (SD), ng/mL & 271.2(249.7) & $358.8(192.5)$ & $<0.01$ \\
\hline CRP, mean (SD), mg/L & $6.3(0.4)$ & $6.9(0.6)$ & 0.7 \\
\hline AST, mean (SD), IU/L & 22.3(13.4) & 23.1(20.2) & 0.6 \\
\hline ALT, mean (SD), IU/L & $24.8(22.4)$ & 24.1(14.3) & 0.6 \\
\hline GGT, mean (SD), IU/L & $49.4(5.6)$ & $35.4(5.7)$ & 0.2 \\
\hline Urea, mean (SD), mg/dL & $5.9(1.8)$ & $5.8(1.2)$ & 0.9 \\
\hline Creatinine $(\mathrm{mg} / \mathrm{dL}$ & $0.9(0.8)$ & $0.8(0.4)$ & 0.4 \\
\hline CK, mean (SD), IU/L & $78.2(6.7)$ & $88.8(9.9)$ & 0.4 \\
\hline LDH, mean (SD), IU/L & 250.4(104.2) & $235.7(90.1)$ & 0.1 \\
\hline
\end{tabular}

Risk factors for persistent post-COVID-19 symptoms 
Table 5 shows risk factors associated with persistent post-COVID-19 symptoms. Many variables showed a significant association in the univariate analysis. A multivariable analysis found that three factors were associated with persistent post-COVID-19 symptoms: female sex, duration of ICU stay, and the onset of ICUAP. In terms of percent change, the odds of persistent post-COVID-19 symptoms in females are $94 \%$ higher than those in males; for a 1-unit increase in the duration of ICU stay, there is an expected $3 \%$ increase in the odds of no clinical resolution; and the odds of persistent post-COVID-19 symptoms for the onset of ICUAP are $73 \%$ higher than the odds for no onset of ICUAP. Among 315 patients who had ICUAP only $34(11 \%)$ had not a pathogen detected and the majority of them were not ventilated. Length of mechanical ventilation was 26 days (15-37 days). The length of ICU stay was 33 days (22.46). Among the 282 patients with confirmed pathogen the mean period of days from ICU admission was 12 days (721 days). Among the 258 patients with confirmed pathogen ,220 were ventilated. $44(20 \%)$ had early VAP and $176(80 \%)$ had late VAP. Gram negatives represented most pathogens isolated were Gram negatives, but $S$. aureus was present in $46(16 \%)$ cases. 4 o patients had polymicrobial pneumonia. A sensitivity analysis introducing the centre variable as a random effect in the mixed-effects multivariable model yielded similar results. 
Table 5

Univariate and multivariate analyses of risk factors associated with persistent post-COVID-19 symptoms at 3-month follow-up

\begin{tabular}{|c|c|c|c|c|c|}
\hline & \multirow{2}{*}{$\begin{array}{l}\text { Univariate } \\
\text { Post-COVID }\end{array}$} & & & \multicolumn{2}{|c|}{ Multivariable( $n=991)$} \\
\hline & & & & OR $(95 \% \mathrm{Cl})$ & $\mathrm{p}$ \\
\hline & Yes & No & $\mathbf{p}$ & & \\
\hline Age, mean (SD), years & $57.85(12.9)$ & $58.74(11.5)$ & 0.3 & & \\
\hline Female sex, n (\%) & $66(25.4)$ & $260(35.6)$ & $\begin{array}{l}< \\
0.01\end{array}$ & $\begin{array}{l}1.94(1.28- \\
2.94)\end{array}$ & 0.002 \\
\hline SOFA, mean (SD) & $4.6(2.8)$ & $(5.2(3.1)$ & 0.01 & & \\
\hline CHF, n (\%) & 28(10.8) & $64(8.8)$ & 0.3 & & \\
\hline Hypertension, n (\%) & $115(44.2)$ & $326(44.7)$ & 0.9 & & \\
\hline COPD, n (\%) & $18(6.9)$ & $63(8.6)$ & 0.4 & & \\
\hline Asthma, n (\%) & $21(8.1)$ & $44(6)$ & 0.2 & & \\
\hline CKD, n (\%) & 10(3.8) & $43(5.9)$ & 0.2 & & \\
\hline Cirrhosis, n (\%) & 0 & $13(1.8)$ & 0.02 & & \\
\hline Mild liver failure, n (\%) & $5(1.9)$ & $14(1.9)$ & 0.9 & & \\
\hline Neurological, n (\%) & $9(3.5)$ & $39(5.3)$ & 0.3 & & \\
\hline Dementia, n (\%) & $1(0.4)$ & $2(0.3)$ & 0.7 & & \\
\hline Autoimmune, n (\%) & $11(4.2)$ & $45(6.2)$ & 0.2 & & \\
\hline Gastrointestinal, n (\%) & $15(5.8)$ & $65(8.9)$ & 0.1 & & \\
\hline Endocrine, n (\%) & $17(6.5)$ & $58(7.9)$ & 0.4 & & \\
\hline
\end{tabular}

Data are mean (SD) or number of patients (\%) for the univariate analysis and estimated ORs (95\% Cls) of the explanatory variables in the persistent post-COVID-19 symptoms group for the multivariable analysis. The OR represents the odds that the presence of persistent post-COVID-19 symptoms will occur given exposure of the explanatory variable, compared to the odds of the outcome occurring in the absence of that exposure; for continuous predictors, the OR represents the increase in odds of the outcome of interest with every one unit increase in the input variable. The pvalue for the multivariable analysis is based on the null hypothesis that all ORs relating to an explanatory variable equal unity (no effect). Abbreviations: OR: Odds ratio. Cl: Confidence interval. SD: Standard deviation. SOFA: Sequential Organ Failure Assessment. CHF: congestive heart failure. COPD: Chronic obstructive pulmonary disease. CKD: Chronic kidney disease. BMI: Body Mass Index. HIV: human immunodeficiency virus. NIVM: Non Invasive Mechanical Ventilation. IMV: Invasive Mechanical Ventilation. ICU: Intensive Care Unit. ECMO: Extracorporeal membrane oxygenation. CRRT: Continuous renal replacement therapy. NMB: Neuromuscular blockade. CPR: Cardiopulmonary resuscitation. ICUAP: Intensive Care Unit acquired pneumonia. ARDS: Acute respiratory distress syndrome. 


\begin{tabular}{|c|c|c|c|c|c|}
\hline Obesity (BMI > $\left.30 \mathrm{~kg} / \mathrm{m}^{2}\right), \mathrm{n}(\%)$ & 98(37.7) & 293(40.1) & 0.5 & & \\
\hline Diabetes Mellitus, n (\%) & $46(17.7)$ & 140(19.2) & 0.6 & & \\
\hline Haematological disease, n (\%) & $13(5)$ & $39(5.3)$ & 0.8 & & \\
\hline Solid cancer, n (\%) & $8(3.1)$ & $21(2.9)$ & 0.8 & & \\
\hline Transplant, n (\%) & $2(0.8)$ & $9(1.2)$ & 0.7 & & \\
\hline HIV, n (\%) & 0 & $4(0.5)$ & 0.5 & & \\
\hline Oxygen requirement, n (\%) & 248(99.2) & 723(99.7) & 0.2 & & \\
\hline NIV, n (\%) & $68(26.5)$ & 280(38.9) & 0 & & \\
\hline IMV, n (\%) & 155(59.6) & $510(70.2)$ & $<.01$ & & \\
\hline Prone, n (\%) & $136(52.3)$ & $431(59.4)$ & 0.04 & & \\
\hline Tracheostomy, n (\%) & $52(20)$ & 260(35.8) & $<.01$ & & \\
\hline $\begin{array}{l}\text { ICU length of stay, mean (SD), } \\
\text { days }\end{array}$ & 14.3(12.2) & 22.2(18.2) & $\begin{array}{l}<.01 \\
0.01\end{array}$ & $\begin{array}{l}1.03(1.01- \\
1.05)\end{array}$ & 0.002 \\
\hline ECMO, n (\%) & $52(2(0.8)$ & $16(2.2)$ & 0.1 & & \\
\hline CRRT, n (\%) & $7(2.7)$ & $47(6.5)$ & 0.02 & & \\
\hline Shock, n (\%) & 132(51.6) & $46(64.3)$ & 0 & & \\
\hline NMB, n (\%) & $124(48.2)$ & $430(59.6)$ & $<.01$ & & \\
\hline Corticosteroids, n (\%) & $207(80.5)$ & $543(75.3)$ & 0.1 & & \\
\hline CPR, n (\%) & $2(0.8)$ & $5(0.7)$ & 0.9 & & \\
\hline
\end{tabular}

Data are mean (SD) or number of patients (\%) for the univariate analysis and estimated ORs (95\% Cls) of the explanatory variables in the persistent post-COVID-19 symptoms group for the multivariable analysis. The OR represents the odds that the presence of persistent post-COVID-19 symptoms will occur given exposure of the explanatory variable, compared to the odds of the outcome occurring in the absence of that exposure; for continuous predictors, the OR represents the increase in odds of the outcome of interest with every one unit increase in the input variable. The pvalue for the multivariable analysis is based on the null hypothesis that all ORs relating to an explanatory variable equal unity (no effect). Abbreviations: OR: Odds ratio. Cl: Confidence interval. SD: Standard deviation. SOFA: Sequential Organ Failure Assessment. CHF: congestive heart failure. COPD: Chronic obstructive pulmonary disease. CKD: Chronic kidney disease. BMI: Body Mass Index. HIV: human immunodeficiency virus. NIVM: Non Invasive Mechanical Ventilation. IMV: Invasive Mechanical Ventilation. ICU: Intensive Care Unit. ECMO: Extracorporeal membrane oxygenation. CRRT: Continuous renal replacement therapy. NMB: Neuromuscular blockade. CPR: Cardiopulmonary resuscitation. ICUAP: Intensive Care Unit acquired pneumonia. ARDS: Acute respiratory distress syndrome. 


\begin{tabular}{|c|c|c|c|c|c|}
\hline \multirow[b]{2}{*}{ ICUAP, n (\%) } & \multicolumn{3}{|l|}{ Univariate } & \multicolumn{2}{|c|}{ Multivariable(n = 991) } \\
\hline & 41(15.9) & 219(30.2) & $\begin{array}{l}< \\
0.01\end{array}$ & $\begin{array}{l}1.73(1.03- \\
2.92)\end{array}$ & 0.03 \\
\hline ARDS, n (\%) & $175(68.4)$ & $564(77.6)$ & $\begin{array}{l}< \\
0.01\end{array}$ & & \\
\hline NTX, n (\%) & $9(3.5)$ & $36(5.0)$ & 0.38 & & \\
\hline COP, n (\%) & $11(4.3)$ & $35(4.9)$ & 0.86 & & \\
\hline PE, n (\%) & $33(13)$ & $64(9)$ & 0.08 & & \\
\hline Delirium, n (\%) & $52(20.1)$ & $182(25.1)$ & 0.1 & & \\
\hline \multicolumn{6}{|c|}{$\begin{array}{l}\text { Data are mean (SD) or number of patients (\%) for the univariate analysis and estimated ORs (95\% } \\
\text { Cls) of the explanatory variables in the persistent post-COVID-19 symptoms group for the } \\
\text { multivariable analysis. The OR represents the odds that the presence of persistent post-COVID-19 } \\
\text { symptoms will occur given exposure of the explanatory variable, compared to the odds of the } \\
\text { outcome occurring in the absence of that exposure; for continuous predictors, the OR represents the } \\
\text { increase in odds of the outcome of interest with every one unit increase in the input variable. The p- } \\
\text { value for the multivariable analysis is based on the null hypothesis that all ORs relating to an } \\
\text { explanatory variable equal unity (no effect). Abbreviations: OR: Odds ratio. Cl: Confidence interval. SD: } \\
\text { Standard deviation. SOFA: Sequential Organ Failure Assessment. CHF: congestive heart failure. } \\
\text { COPD: Chronic obstructive pulmonary disease. CKD: Chronic kidney disease. BMI: Body Mass Index. } \\
\text { HIV: human immunodeficiency virus. NIVM: Non Invasive Mechanical Ventilation. IMV: Invasive } \\
\text { Mechanical Ventilation. ICU: Intensive Care Unit. ECMO: Extracorporeal membrane oxygenation. } \\
\text { CRRT: Continuous renal replacement therapy. NMB: Neuromuscular blockade. CPR: Cardiopulmonary } \\
\text { resuscitation. ICUAP: Intensive Care Unit acquired pneumonia. ARDS: Acute respiratory distress } \\
\text { syndrome. }\end{array}$} \\
\hline
\end{tabular}

\section{Discussion}

The present study analysed a multicentre cohort of patients admitted to the ICU with COVID-19. Few data are currently available on the follow-up of survivor patients with COVID-19 after discharge. Interestingly, we found that more than $70 \%$ of patients discharged from the ICU had persistent post-COVID-19 symptoms after three months passed since hospital discharge; however, the hospital re-admission rate within this period remained low and only $15 \%$ needed to visit the emergency department. There was also a poor correlation between abnormal radiologic findings and persistent post-COVID-19 symptoms in critically ill patients after three months passed since hospital discharge. Factors associated with persistent post-COVID-19 symptoms included female sex, duration of ICU stay, and the onset of ICUAP.

With respect to the burden of follow-up on the healthcare system, x-rays and CT scans were performed in 4 of 10 ICU-admitted patients after three months since hospital discharge. As expected, normal $x$-rays were frequent in patients with good clinical resolution; interstitial patterns were more often seen in chest CT scans of patients with persistent post-COVID-19 symptoms. Recently, patients with previously undiagnosed fibrotic lung abnormalities have been reported to face the possibility of ARDS onset [19]. In a cohort of 114 survivors of severe COVID-19 monitored for six months, chest CT scans revealed fibrotic- 
like changes in the lungs in more than one-third of cases [20]. In contrast, only $2.5 \%$ of our cohort presented with an interstitial lung pattern. In our view, an important issue is to determine patients' need for resources after hospital discharge. More than $15 \%$ of our cohort patients discharged from the ICU required oxygen; yet at the time of writing this manuscript, only a minority $(5 \%)$ continued receiving supplementary oxygen at home. Interestingly, nebulizers were less frequently used than supplementary oxygen after hospital discharge. To the best of our knowledge, this is a novel finding. Few data are, however, available regarding additional therapy in patients with COVID-19 discharged from the ICU; this is a point that warrants further exploration. Investigators Banerjee et al. [21] followed 621 discharged patients receiving oxygen at home and reported a 30-day hospital re-admission rate of $8.5 \%$.

Readmission rate in our cohort was much lower than that in that study (3.1\%), although more patients (15\%) visited the emergency department after discharge.

The symptoms most frequently observed in patients with post-COVID-19 included dyspnoea, asthenia, and weakness. After analysis of the therapy provided, we found that oxygen therapy was provided significantly more often to patients with persistent post-COVID-19 symptoms. In a previous study in China, patients monitored for three months after hospital discharge presented with considerable radiologic and physiologic abnormalities [22]. In another Chinese study[23] including over 1000 patients, survivors of COVID-19 presented with fatigue, sleep difficulties and anxiety or depression at 6-month follow-up. However, as in the previous study, no detailed data about the functional status of the patients were provided. In our study, we did not perform any analysis related to depression or anxiety.

A strength of our research includes the prospective follow-up of a detailed list of lung function parameters. Soriano et al. [24] recently wrote an editorial suggesting more studies be done in clinical research assessing the post-COVID-19 condition. Our study integrates an extremely sizeable cohort and evaluates a relevant subgroup of the population, i.e., critically ill patients. A French study found that patients with COVID-19 had some symptoms not previously present before their disease[25]. These findings can complement our report, as most of the patients included in our cohort had a critically ill condition. Moreover, in our cohort, ICU-admitted patients with no clinical resolution had worse forced expiratory volume in the first second (FEV1) than those who did not present with persistent post-COVID19 symptoms. Some studies-the majority from China and some from Europe, with limited patient samples-have also found substantial differences in LFT; however, most patients included were not critically ill [26][27]. In addition to FEV1, FEV1/FVC and DLCO presented significant differences. In a study done in Sweden, investigators Ekbom et al. [28] found that over half of patients with COVID-19 treated in the ICU had impaired lung function during follow-up, suggesting further follow-up studies including DLCO. In their cohort, a mean DLCO of $62 \%$ was reported as predicted amongst those with abnormal DLCO. These figures are like ours. We observed a significant correlation between abnormal DLCO and poor health post-COVID-19 in our cohort. The presence of decreased DLCO might reflect microvascular or alveolar capillary damage and be expected in patients with no clinical resolution[29]. Very little is known about the pathophysiology of poor health post-COVID-19. COVID-19 causes lung damage due to a marked inflammatory response to the virus. As is known, the disease may damage endothelial cells in the lung parenchyma. Therefore, identifying pathways may prove as a key point in determining this damage. 
Ward et al. [30] found that increased plasma levels of von Willebrand factor antigen (VWF:Ag) and procoagulant factor VIII (FVIII) were seen in patients with SARS-CoV-2 infection. In our cohort, we found only elevated levels of fibrinogen in patients with persistent post-COVID-19 symptoms; this observation could help determine the role of endothelial activation in pathophysiology of the disease.

Additionally, we aimed to determine an association between ventilatory parameters in patients with invasive mechanical ventilation and persistent post-COVID-19 symptoms at the 3-month follow-up since hospital discharge. Our assessment of different respiratory parameters such as $\mathrm{PaO}_{2} / \mathrm{FiO}_{2}$ ratio and $\mathrm{pCO}_{2}$ showed a significant correlation across two variables: compliance at the time of intubation and $\mathrm{PCO}_{2}$ at day 3 . Both parameters clearly reflect the damage caused to the respiratory system by a COVID19 infection. It is interesting that compliance, albeit not the $\mathrm{PaO}_{2} / \mathrm{FiO}_{2}$ ratio, was a predictor of persistent post-COVID-19 symptoms. A recent study found that median time to intubation was twice as long in the very-low compliance group than in the low-normal compliance group [31]. Reported higher levels of $\mathrm{PaCO} 2$ in patients in the very-low lung compliance group in that study correlated strongly with our findings. Furthermore, some authors [32] have suggested that compliance in COVID-19-related ARDS is higher in non-COVID-19-related ARDS; our finding could find explanation by the fact that patients with low compliance were those with more severe ARDS. Recently, Gonzalez et al. found that abnormal results were present in CT scans of more than two-thirds of patients with COVID-19-related ARDS[33].

The last and perhaps most important finding of our study is the identification of independent risk factors for persistent post-COVID-19 symptoms.

The first risk factor is female sex. This is an intriguing finding, given that male patients are more widely reported to be admitted to the ICU[34][35] for COVID-19. Further, a systematic review found that COVID-19 may be associated with worse outcomes in males than in females [36]. Whilst most ICU-admitted patients in our cohort were male (67\%), more female patients had persistent post-COVID-19 symptoms at 3-month follow-up. The PHOSP-COVID study conducted in the United Kingdom observed that $70 \%$ had not fully recovered a mean follow-up period of five months after hospital discharge, with women being more than men [37].

In addition to female patients, another group who presented with poor recovery included critically ill patients with a longer duration of ICU stay. After applying for adjustment, we found that tracheostomy was not an independent risk factor for poor recovery. This finding challenges the recommendation that early tracheostomy may reduce recovery time in critical COVID-19[38].

Lastly, the onset of pneumonia during the ICU stay proved to be an independent risk factor fat 3-month follow-up. This is a very especially important finding given the high incidence of nosocomial pneumonia in critically ill patients, especially than those that needed invasive mechanical ventilation. This finding stresses the importance of the prevention ICUAP in COVID-19 critically ill patients. The hypothesis behind this finding is that a second hit (ICUAP) after COVID-19 increased lung damage and consequently increased the risk of respiratory symptoms persistence at follow-up. Some multicentre, European 
manuscripts suggest that ventilator-associated lower respiratory tract infections (VA-LRTI) were more frequent in patients with COVID-19 than in patients admitted to the ICU with another virus (influenza) or in patients without viral infections [39]. To the best of our knowledge, our finding has not been reported elsewhere, and other studies should be carried out to confirm or refute it.

Our study has several limitations. Samples obtained from the centres may not be representative, given that hospital units selected all had the research resources necessary to participate. We included an acceptable number of variables for follow-up analysis; however, certain functional tests were not recorded, including the six-minute walk test (6MWT), an excellent tool for assessing sub-maximal exercise aerobic capacity and endurance. We preferred to determine LFT and imaging, as detailed extensively in this manuscript, and felt that the 6MWT might not be reproducible as a measure for oxygen desaturation. A strength of this manuscript is the availability of many data points from the acute period and including data of day 1 and day 3 to determine risk factors of Long-Covid 19 syndrome.

\section{Conclusion}

In conclusion, we evaluated many critically ill patients with COVID-19 after three months passed since hospital discharge. Persistent post-COVID-19 symptoms occurred in more than two-thirds of patients; however, the hospital readmission rate remained low. There was not clinical association between such symptoms and persistently abnormal chest x-rays. Also, more than $10 \%$ of these patients still required oxygen at home. Female sex, duration of ICU stay, and the onset of ICUAP were independent risk factors for persistent post-COVID-19 symptoms in critically ill patients at 3-month follow-up. Prevention of ICUAP could have beneficial effects in poor health post-Covid 19 syndrome.

\section{Declarations}

- Ethics approval and consent to participate: The study received approval by the institution's Internal Review Board (Comité Ėtic d'Investigació Clínica, registry number HCB/2020/0370).

- Consent for publication: All authors listed on the title page have read the manuscript, attest to the validity, and legitimacy of the data and its interpretation, and agree to its submission to Critical Care.

- Availability of data and material. Data and material are available.

- Competing interests. The authors declare that they have no competing interests.

- Funding: CIBERESUCICOVID. CIBER enfermedades respiratorias

- Authors' contributions. IML and AT designed the study. All other authors contributed to data acquisition and approved the manuscript.

- Acknowledgements. To all the patients and families

\section{References}


1. Rello J, Storti E, Belliato M, Serrano R. Clinical phenotypes of SARS-CoV-2: implications for clinicians and researchers. Eur Respir J [Internet]. 2020;55:2001028. Available from:

http://erj.ersjournals.com/lookup/doi/10.1183/13993003.01028-2020.

2. Kolb M, Dinh-Xuan AT, Brochard L. Guideline-directed management of COVID-19: Do's and Don'ts. Eur Respir J [Internet]. 2021;57:2100753. Available from: http://erj.ersjournals.com/lookup/doi/10.1183/13993003.00753-2021.

3. Blake A, Collins D, O'Connor E, Bergin C, McLaughlin AM, Martin-Loeches I. Clinical and biochemical characteristics of patients admitted to ICU with SARS-CoV-2. Med Intensiva [Internet]. 2020;44:58990. Available from: https://linkinghub.elsevier.com/retrieve/pii/S0210569120301662.

4. Martin-Loeches I, Arabi Y, Citerio G. If not now, when? A clinical perspective on the unprecedented challenges facing ICUs during the COVID-19 pandemic. Intensive Care Med [Internet]. 2021;47:58890. Available from: https://link.springer.com/10.1007/s00134-021-06404-3.

5. Esperatti M, Ferrer M, Theessen A, Liapikou A, Valencia M, Saucedo LM, et al. Nosocomial pneumonia in the intensive care unit acquired by mechanically ventilated versus nonventilated patients. Am J Respir Crit Care Med [Internet]. 2010 [cited 2014 Aug 30];182:1533-9. Available from: http://www.ncbi.nlm.nih.gov/pubmed/20693381.

6. Vaes AW, Goërtz YMJ, Van Herck M, Machado FVC, Meys R, Delbressine JM, et al. Recovery from COVID-19: a sprint or marathon? 6-month follow-up data from online long COVID-19 support group members. ERJ Open Res [Internet]. 2021;7:00141-2021. Available from: http://openres.ersjournals.com/lookup/doi/10.1183/23120541.00141-2021.

7. D'Cruz RF, Waller MD, Perrin F, Periselneris J, Norton S, Smith L-J, et al. Chest radiography is a poor predictor of respiratory symptoms and functional impairment in survivors of severe COVID-19 pneumonia. ERJ Open Res [Internet]. 2021;7:00655-2020. Available from: http://openres.ersjournals.com/lookup/doi/10.1183/23120541.00655-2020.

8. Townsend L, Dowds J, O'Brien K, Martin-Loeches I, Nadarajan P, Bannan C. More on Persistent Poor Health Post-COVID-19 Is Not Associated with Respiratory Complications or Initial Disease Severity. Ann Am Thorac Soc [Internet]. 2021;AnnalsATS.202101-073LE. Available from: https://www.atsjournals.org/doi/10.1513/AnnalsATS.202101-073LE.

9. Salamanna F, Veronesi F, Martini L, Landini MP, Fini M. Post-COVID-19 Syndrome: The Persistent Symptoms at the Post-viral Stage of the Disease. A Systematic Review of the Current Data. Front Med [Internet]. 2021;8. Available from:

https://www.frontiersin.org/articles/10.3389/fmed.2021.653516/full.

10. Townsend L, Dyer AH, Jones K, Dunne J, Mooney A, Gaffney F, et al. Persistent fatigue following SARS-CoV-2 infection is common and independent of severity of initial infection. Madeddu G, editor. PLoS One [Internet]. 2020;15:e0240784. Available from: https://dx.plos.org/10.1371/journal.pone.0240784.

11. Bangash MN, Owen A, Alderman JE, Chotalia M, Patel JM, Parekh D. COVID-19 recovery: potential treatments for post-intensive care syndrome. Lancet Respir Med [Internet]. 2020;8:1071-3. Available 
from: https://linkinghub.elsevier.com/retrieve/pii/S2213260020304574.

12. Parker AJ, Humbir A, Tiwary P, Mishra M, Shanmugam M, Bhatia $K$, et al. Recovery after critical illness in COVID-19 ICU survivors. Br J Anaesth [Internet]. 2021;126:e217-9. Available from: https://linkinghub.elsevier.com/retrieve/pii/S0007091221001628.

13. Estella Á, Garcia Garmendia JL, de la Fuente C, Machado Casas JF, Yuste ME, Amaya Villar R, et al. Predictive factors of six-week mortality in critically ill patients with SARS-CoV-2: A multicenter prospective study. Med Intensiva [Internet]. 2021; Available from: https://linkinghub.elsevier.com/retrieve/pii/S0210569121000334.

14. Adeloye D, Elneima O, Daines L, Poinasamy K, Quint JK, Walker S, et al. The long-term sequelae of COVID-19: an international consensus on research priorities for patients with pre-existing and newonset airways disease. Lancet Respir Med [Internet]. 2021; Available from: https://linkinghub.elsevier.com/retrieve/pii/S2213260021002861.

15. Vincent J-L, Moreno R, Takala J, Willatts S, De Mendonça A, Bruining H, et al. The SOFA (Sepsisrelated Organ Failure Assessment) score to describe organ dysfunction/failure. Intensive Care Med [Internet]. 1996;22:707-10. Available from: http://link.springer.com/10.1007/BF01709751.

16. Martin-Loeches I, Torres A, Povoa P, Zampieri FG, Salluh J, Nseir S, et al. The association of cardiovascular failure with treatment for ventilator-associated lower respiratory tract infection. Intensive Care Med [Internet]. 2019;45:1753-62. Available from: http://www.ncbi.nlm.nih.gov/pubmed/31620836.

17. Acute Respiratory Distress Syndrome. JAMA [Internet]. 2012;307. Available from: http://jama.jamanetwork.com/article.aspx?doi=10.1001/jama.2012.5669.

18. Bestall JC, Paul EA, Garrod R, Garnham R, Jones PW, Wedzicha JA. Usefulness of the Medical Research Council (MRC) dyspnoea scale as a measure of disability in patients with chronic obstructive pulmonary disease. Thorax [Internet]. 1999;54:581-6. Available from: https://thorax.bmj.com/lookup/doi/10.1136/thx.54.7.581.

19. Putman RK, Hunninghake GM, Dieffenbach PB, Barragan-Bradford D, Serhan K, Adams U, et al. Interstitial Lung Abnormalities Are Associated with Acute Respiratory Distress Syndrome. Am J Respir Crit Care Med [Internet]. 2017;195:138-41. Available from: http://www.atsjournals.org/doi/10.1164/rccm.201604-0818LE.

20. Han X, Fan Y, Alwalid O, Li N, Jia X, Yuan M, et al. Six-month Follow-up Chest CT Findings after Severe COVID-19 Pneumonia. Radiology [Internet]. 2021;299:E177-86. Available from: http://pubs.rsna.org/doi/10.1148/radiol.2021203153.

21. Banerjee J, Canamar CP, Voyageur C, Tangpraphaphorn S, Lemus A, Coffey C, et al. Mortality and Readmission Rates Among Patients With COVID-19 After Discharge From Acute Care Setting With Supplemental Oxygen. JAMA Netw Open [Internet]. 2021;4:e213990. Available from: https://jamanetwork.com/journals/jamanetworkopen/fullarticle/2778078.

22. Zhao Y, Shang Y, Song W, Li Q, Xie H, Xu Q, et al. Follow-up study of the pulmonary function and related physiological characteristics of COVID-19 survivors three months after recovery. 
EClinicalMedicine [Internet]. 2020;25:100463. Available from:

https://linkinghub.elsevier.com/retrieve/pii/S2589537020302078.

23. Huang C, Huang L, Wang Y, Li X, Ren L, Gu X, et al. 6-month consequences of COVID-19 in patients discharged from hospital: a cohort study. Lancet [Internet]. 2021;397:220-32. Available from: https://linkinghub.elsevier.com/retrieve/pii/S0140673620326568.

24. Soriano JB, Waterer G, Peñalvo JL, Rello J. Nefer, Sinuhe and clinical research assessing post COVID19 condition. Eur Respir J [Internet]. 2021;57:2004423. Available from:

http://erj.ersjournals.com/lookup/doi/10.1183/13993003.04423-2020.

25. Morin L, Savale L, Pham T, Colle R, Figueiredo S, Harrois A, et al. Four-Month Clinical Status of a Cohort of Patients After Hospitalization for COVID-19. JAMA [Internet]. 2021;325:1525. Available from: https://jamanetwork.com/journals/jama/fullarticle/2777787.

26. Anastasio F, Barbuto S, Scarnecchia E, Cosma P, Fugagnoli A, Rossi G, et al. Medium-term impact of COVID-19 on pulmonary function, functional capacity and quality of life. Eur Respir J [Internet]. 2021;2004015. Available from: http://erj.ersjournals.com/lookup/doi/10.1183/13993003.040152020.

27. Huang Y, Tan C, Wu J, Chen M, Wang Z, Luo L, et al. Impact of coronavirus disease 2019 on pulmonary function in early convalescence phase. Respir Res [Internet]. 2020;21:163. Available from: https://respiratory-research.biomedcentral.com/articles/10.1186/s12931-020-01429-6.

28. ÖI EERF, IM E L, M L, S R, et al. Impaired diffusing capacity for carbon monoxide is common in critically ill Covid-19 patients at four months post-discharge. Respir Med [Internet]. 2021;106394. Available from: https://linkinghub.elsevier.com/retrieve/pii/S0954611121001001.

29. O'Donnell JS, Peyvandi F, Martin-Loeches I. Pulmonary immuno-thrombosis in COVID-19 ARDS pathogenesis. Intensive Care Med [Internet]. 2021; Available from: http://www.ncbi.nlm.nih.gov/pubmed/34052905.

30. Ward SE, Curley GF, Lavin M, Fogarty H, Karampini E, McEvoy NL, et al. Von Willebrand factor propeptide in severe coronavirus disease 2019 (COVID-19): evidence of acute and sustained endothelial cell activation. Br J Haematol [Internet]. 2021;192:714-9. Available from: https://onlinelibrary.wiley.com/doi/10.1111/bjh.17273.

31. http://medrxiv.org/content/early/2021/01/28/2021.01.26.21250492.abstract.

32. Gattinoni L, Coppola S, Cressoni M, Busana M, Rossi S, Chiumello D. COVID-19 Does Not Lead to a "Typical" Acute Respiratory Distress Syndrome. Am J Respir Crit Care Med [Internet]. 2020;201:1299300. Available from: https://www.atsjournals.org/doi/10.1164/rccm.202003-0817LE.

33. González J, Benítez ID, Carmona P, Santisteve S, Monge A, Moncusí-Moix A, et al. Pulmonary Function and Radiologic Features in Survivors of Critical COVID-19. Chest [Internet]. 2021; Available from: https://linkinghub.elsevier.com/retrieve/pii/S0012369221004645.

34. Grasselli G, Zangrillo A, Zanella A, Antonelli M, Cabrini L, Castelli A, et al. Baseline Characteristics and Outcomes of 1591 Patients Infected with SARS-CoV-2 Admitted to ICUs of the Lombardy Region, 
Italy. JAMA - J Am Med Assoc [Internet]. American Medical Association; 2020 [cited 2021 Mar 29];323:1574-81. Available from: https://jamanetwork.com/.

35. Guan W, Ni Z, Hu Y, Liang W, Ou C, He J, et al. Clinical Characteristics of Coronavirus Disease 2019 in China. N Engl J Med. 2020;1-13.

36. Lakbar I, Luque-Paz D, Mege J-L, Einav S, Leone M. COVID-19 gender susceptibility and outcomes: A systematic review. PLoS One [Internet]. 2020;15:e0241827. Available from:

http://www.ncbi.nIm.nih.gov/pubmed/33141872.

37. Evans RA, McAuley H, Harrison EM, Shikotra A, Singapuri A, Sereno M, et al. Physical, cognitive and mental health impacts of COVID-19 following hospitalisation - a multi-centre prospective cohort study. medRxiv [Internet]. 2021;2021.03.22.21254057. Available from:

http://medrxiv.org/content/early/2021/03/24/2021.03.22.21254057.abstract.

38. Arnold J, Gao CA, Malsin E, Todd K, Argento AC, Cuttica M, et al. Outcomes of Percutaneous Tracheostomy for Patients with SARS-CoV-2 Respiratory Failure. medRxiv Prepr Serv Heal Sci [Internet]. 2021; Available from: http://www.ncbi.nlm.nih.gov/pubmed/33655275.

39. Rouzé A, Martin-Loeches I, Povoa P, Makris D, Artigas A, Bouchereau M, et al. Relationship between SARS-CoV-2 infection and the incidence of ventilator-associated lower respiratory tract infections: a European multicenter cohort study. Intensive Care Med [Internet]. 2021;47:188-98. Available from: http://www.ncbi.nIm.nih.gov/pubmed/33388794.

\section{Figures}

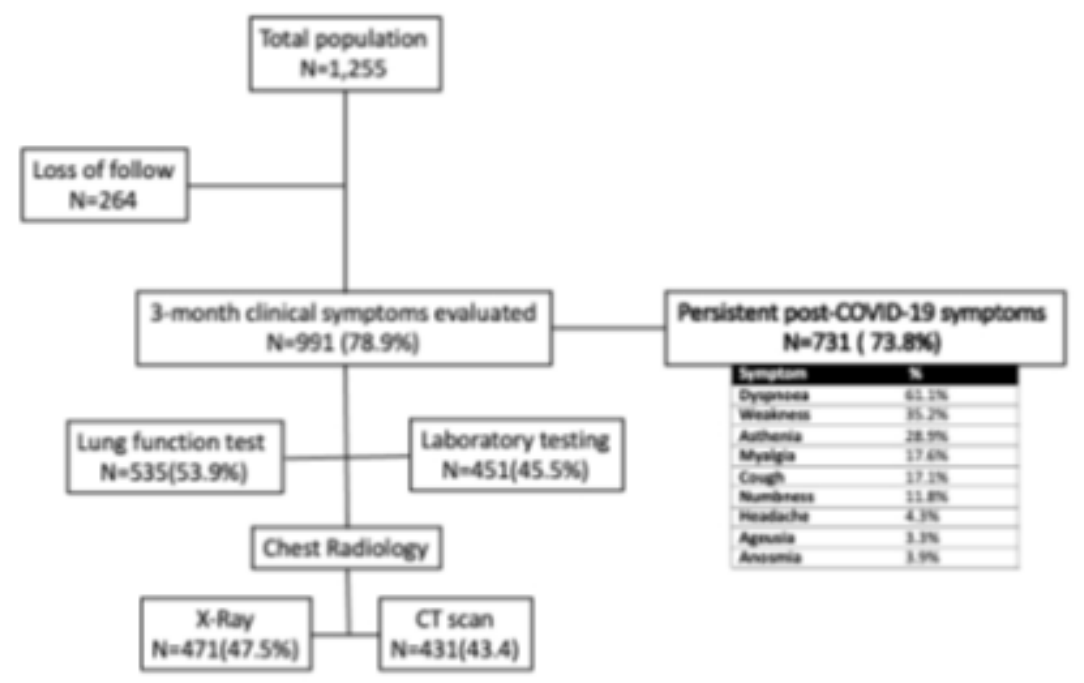

Figure 1. Flow chart and the percentage of patients with persistent post-COVID-19 symptoms.

\section{Figure 1}


See image above for figure legend

\section{Supplementary Files}

This is a list of supplementary files associated with this preprint. Click to download.

- ColaboradoresCIBERESUCICOVID.docx 